\title{
The Frequency of CA15-3, CA125, CA19-9 in Patients With Hepatitis B and C
}

\author{
Somayeh Yeganeh-Amirkande ${ }^{1}$; Mehdi Assmar ${ }^{1, *}$; Fariborz Mansour-Ghanaei ${ }^{2}$; Amir \\ Mozafari-Noor ${ }^{3}$ \\ ${ }^{1}$ Department of Microbiology, Lahijan Branch, Islamic Azad University, Lahijan, IR Iran \\ ${ }^{2}$ Gastrointestinal and Liver Diseases Research Center, Guilan University of Medical Sciences, Rasht, IR Iran \\ 3 Department of Microbiology, Iran University of Medical Sciences, Tehran, IR Iran \\ *Corresponding author: Mehdi Assmar, Department of Microbiology, Lahijan Branch, Islamic Azad University, Lahijan, IR Iran. E-mail: mehdiassmar@yahoo.com
}

Received: February 15, 2014; Accepted: March 8, 2014

Background: Hepatitis B is considered as a cause for serum hepatitis which may lead to liver cells cancer. Hepatitis $\mathrm{C}$ is major cause of chronic hepatitis in developed countries. Information about tumor markers in patients with HBV and HCV in Iran population is limited. Objectives: Therefore, this study aimed to determine the role of tumor markers CA15-3, CA125, CA19-9 in patients with hepatitis B and C who refer to Guilan Liver and Digestive Disease Research Center.

Patients and Methods: In this descriptive cross sectional study on serum samples from 80 patients with hepatitis B and C at Guilan Liver and Digestive Disease Research Center has been conducted from October 2012 to October 2013 in terms of listed tumor markers via ELISA method.

Results: The findings showed that no increases in serum levels of tumor marker CA19-9 has been seen in patients with hepatitis. In patients with hepatitis $\mathrm{B}$, there was an increasing at levels of tumor marker $\mathrm{CA15}-3(\mathrm{P}=0.04)$. Also in patients with hepatitis $\mathrm{C}$, increasing in the tumor marker CA125 were observed $(\mathrm{P}=0.02)$.

Conclusions: The study showed that the tumor marker CA15-3 and tumor marker CA125 was high respectively in hepatitis B and hepatitis $\mathrm{C}$, but this increasing is not for malignancy, but further studying seemed to be necessary because of low size of samples to find the reasons of the increasing.

Keywords: Frequency; Tumor markers; Hepatitis B; Hepatitis C; ELISA

\section{Background}

Hepatitis B (hepatitis B virus) is classified as DNA-based hepatic viruses transferring through body fluid, blood sexual intercourse and in the last months of gestation through mother to the fetus. There is no relationship between having this disease and age, season or gender. This disease has a 3 months incubation period. In 5 - 10\% of patients it will turn to chronic disease and sometimes leads to the liver malignancy [1]. Up to 350 million people all over the world suffer from chronic hepatitis B which this figure accounts for about $7 \%$ of total population worldwide $[2,3]$.

Prevalence of this disease in Middle East countries like Iran is in medium range. In a study performed in Iran, $1.7 \%$ of the population had this infection [4]. Infection of people to $\mathrm{HCV}$ (hepatitis $\mathrm{C}$ virus) as an infection transmitting through blood potentially may occur through sexual intercourse or during the gestation period. Worldwide propagation of HCV based on finding antibody against the serum in the blood of infected people has been estimated as 3\% and is more than 170 million people with HCV [1]. Hepatitis B and C due to formation of immune complex by virus antigen or antibody in the glomerular basement membrane, leads to production of membranous glomerulonephritis and membrane proliferation. In extreme cases, severity of hepatic disease will surpass the symptoms of hepatic complication. Mixed cryoglobulinemia, due to production of antibody with particular physical properties leading to precipitation in low temperature was reported in hepatitis B and currently in HCV [5]. Tumor markers are biomolecules that share the structure of proteins and hormones found in abnormal blood, urine and tissue of patients with all kinds of cancer. Measurement of a tumor marker in most cases is limited to the diagnosis, since most tumor markers do not act specifically and will increase in number within the patients without cancer. In most cancers, it is possible that several tumor markers increase, therefore study on them is of diagnosing value. Once a table of tumor markers related to a specific cancer is built, their diagnosing value will increase. All the tumor markers have less than 100\% sensitivity and specificity, therefore it can be stated that no tumor markers are specific to an organ and are not specific to the cancer [6]. Tumor markers can be used

Copyright (C) 2015, Zahedan University of Medical Sciences. This is an open-access article distributed under the terms of the Creative Commons Attribution-NonCommercial 4.0 International License (http://creativecommons.org/licenses/by-nc/4.0/) which permits copy and redistribute the material just in noncommercial usages, provided the original work is properly cited. 
for one of five purposes 1) screening for the presence of cancer 2) monitoring the course of cancer in patients 3) diagnosis of cancer or of a specific type of cancer 4) determining the prognosis in patients 5) determining stage of cancer. High levels of tumor markers in cancer patients can be used to help determine the extent at which the cancer has spread to other tissues and organ. Tumor markers studied in this experiment include CA19-9 (Carbohydrate antigen 19-9), CA125 (Cancer antigen 125), and CA15-3 (Cancer antigen 15-3).

CA125 is a modified antigen with Mullerian fetus cells. This antigen has a semi-mucin glycoprotein structure and is considered as a major marker in ovary non-mucin tumors and endometriosis [7, 8]. CA19-9 is a glycoprotein expressed by several epithelial cancers. It is considered as the marker of pancreatic cancer and sometimes as the marker of hepatocellular carcinoma, colon, and rectum cancers. Elevated CA19-9 serum levels have been observed in patients with gastric adenocarcinoma $[8,9]$. CA15-3 is considered as the biomarker of breast cancer. It has a glycoprotein structure which in most carcinomas including adenocarcinoma. An increased CA15-3 value can indicate lung, ovarian, liver, or stomach cancer [7-9].

Many studies have shown that elevation in tumor markers appear to be useful in the diagnosis of hepatitis and gastrointestinal tract. CA19-9, CA125, CA15-3 may elevate in other malignancies as well as in benign conditions. The clinical significance of elevated tumor markers in patients with HBV and HCV are not well defined.

\section{Objectives}

The objective of study was to determine the significance and implications of elevated CA19-9, CA125, CA15-3 levels in the serum. Previous studies showed the relationship between the elevated some of tumor markers and hepatitis. This study based an included analysis of demographic factors (gender, age), histological and virological factors. We investigated the correlation between CA19-9, CA125, CA15-3 serum levels and severity of the liver disease and in order to evaluate whether elevated CA19-9, CA125, CA153 serum levels could depend on the viral infection or can be considered a non-common event.

\section{Patients and Methods}

\subsection{Patients}

This descriptive cross sectional study performed from October 2012 to October 2013,197 patients with hepatitis B and C referred to Guilan Research Center for Digestion and Liver. Of 197 patients, 117 patients receiving drugs related to hepatitis including interferon- , ribavirin, lamivudine, etc. were emitted from the project. The study was conducted on 80 untreated patients with hepatitis B and C, 54 of which were males and 26 were females with ages ranging from 18 to 57 years. Firstly, patients with hepatitis referring to Guilan Research Center for Digestion and Liver were introduced to the project after giving consent. Research questionnaire was including the demographic, endoscopy, liver biopsy information and the last laboratory findings of patients and it was completed through interview. Patients history was included their age, gender and BMI (body mass index).

Inclusion Criteria: 1) hepatitis C virus antibody (HCV $\mathrm{Ab})$ by AxSYM and confirmed by real-time polymerase chain reaction (RT-PCR) positive 2) positive HBs Ag 3) no history of antiviral therapy 4) no evidence of HCC with regular abdominal sonography or Magnetic Resonance Imaging (MRI) or other imaging. Exclusion criteria: 1) Patients who had received drug-related hepatitis (subcutaneous injection of drugs such as interferon-alpha in combination with diet and oral ribavirin, lamivudine 2) patients who did not consent to continue.

\subsection{Limitations of the Study}

Also patients were not totally comfortable to release their medical history to us. Some people are upset and angry because of the lack of sufficient awareness of the problems of their patients were not willing to cooperate.

\subsection{Ethical Considerations}

Patients were informed of the plan and the fact that their medical record will be kept confidential.

\subsection{Laboratory Investigations}

In all the patients introduced in the study, blood was taken before starting any medication. The blood samples were placed in $4^{\circ} \mathrm{C}$ in laboratory conditions until clotting occurred. Then the patient's serums were separated by centrifuge and were placed in refrigerator in $20^{\circ} \mathrm{C}$ before analyzing. During examination, a Complete Blood Count (CBC) of each patient was defined. CBC was performed using a SYSMEX K1000. Prothrombin time and concentration were measured using a Bench Electronic coagulator. In addition, serum levels of bilirubin, alanine aminotransferase (ALT), asparagine aminotransferase (AST) and alkaline phosphatase (ALP) were measured using a BM Hitachi 711 Chemistry Analyzer. In this study use from Pars azmoon kits for enzyme test. A serum sample of each patient was tested for hepatitis B and C. Measurement of HBs Ag, IgM antibodies to hepatitis B core antigen ( $\mathrm{Hbc}$ Ab IgM), HBc Ab, hepatitis B surface antigen (HBs $\mathrm{Ab}$ ) and HCV Ab were done by IM.E.SA BT 3000 automated immunoassay analyzer, based on quantitative enzyme micro particle immunoassay. After defining the positive cases of hepatitis, they were analyzed in respect of tumor markers CA19-9, CA125, and CA15-3 using quantitative ELISA. To perform ELISA tests by Xema kits from Russia (Kit-code M12 and ZFP kits (Kit-code ZK222, ZK223, ZK224, ZK225) produced by Iranian company of Zist Faravard Pars. 


\subsection{Statistical Analysis}

Analysis of data collected in the questionnaire was analyzed using SPSS-16 (Statistical Package for Social Sciences) with fisher test. In the analysis of the results $\mathrm{P}<0.05$ was significant.

\section{Results}

Of 80 patients, 49 cases were infected with hepatitis B, in which 20 cases (40.8\%) were female and 29 (59.2\%) were male. Thirty one patients were infected with hepatitis $\mathrm{C}$, in which 6 cases (19.4\%) were female and 25 (80.6\%) were male. The prevalence of both hepatitis among males is higher than that found in females (Figure 1).

The patients were in the age group of 18 - 57 years. The highest increase in hepatitis B was related to the 48 - 57 years age groups with a frequency of 16 persons (32.7\%). And the highest increase in hepatitis $C$ was belonged to the 28 - 37 age groups with a frequency of 11 persons (35.5\%), (Figure 2). In the present study it was shown that there is no significant relationship between tumor marker CA19-9 and patients with hepatitis B and C.

It is also found that for patients with hepatitis B there is a significant increase in tumor marker CA15-3 level, and a statistically significant relationship between hepatitis $\mathrm{B}$ and the level of these tumor markers was observed $(P=0.04)$. In patients with hepatitis $C$, a significant increase in tumor marker CA125 was observed that based. On statistical results there is also a meaningful relationship between hepatitis $\mathrm{C}$ and the level of these tumor markers $(\mathrm{P}=0.02)$.

The results showed no significant relationship between age and tumor markers. Also there was no significant relationship between this disease and parameters such as job, sex, albumin, ferritin, ALP, PT and tumor markers. However, in this study, the significant relationship between liver enzymes (ALT, AST) and level increase of tumor markers CA15-3 and CA125 was observed $(P=0.03)($ Table 1$)$.

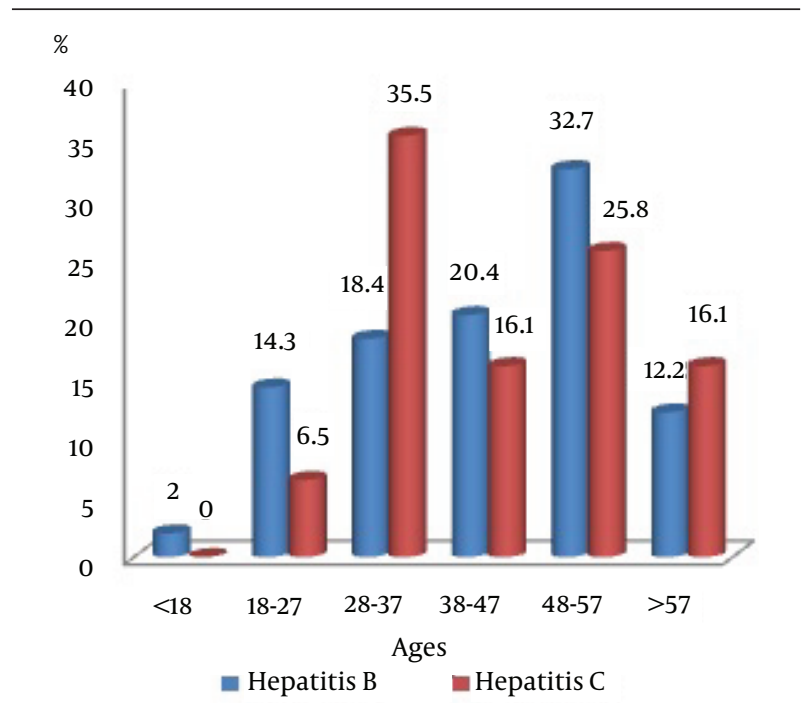

Figure 1. Frequency vs. Sex for Two Groups of Patients Infected by Hepatitis $B$ and $C$

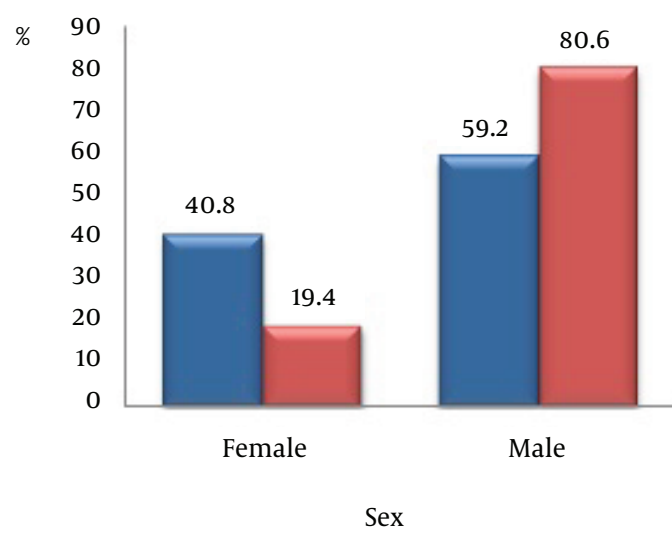

Hepatitis B $\mathbf{m}$ Hepatitis C

Figure 2. Frequency vs. Age for Two Groups of Patients Infected by Hepatitis B and C

Table 1. Tumor Markers Frequency in Patients Infected by Hepatitis B and C vs.

\begin{tabular}{lccc}
\hline Tumor Marker & Hepatitis BN, \% & Hepatitis CN, \% & P-Value \\
\hline CA15-3 & $35(71.44)$ & & 0.04 \\
\hline Normal & $14(28.56)$ & $31(100)$ & \\
\hline Abnormal & $49(100)$ & $31(100)$ & 0.02 \\
\hline Total & & & \\
\hline CA125 & $46(93.9)$ & $22(70.9)$ & NS \\
\hline Normal & $3(6.1)$ & $9(29.1)$ & \\
\hline Abnormal & $49(100)$ & $31(100)$ & \\
\hline Total & & $29(93.5)$ & \\
\hline CA19-9 & $48(98)$ & $2(6.5)$ & \\
\hline Normal & $1(2)$ & $31(100)$ & \\
\hline Abnormal & $49(100)$ & & \\
\hline Total & &
\end{tabular}




\section{Discussion}

In this study, tumor markers CA15-3, CA125 and CA19-9 were studied as non-invasive markers of patients with hepatitis B and C. Also in this study a significant relationship between tumor marker CA15-3 level and hepatitis infection was observed. And there was a significant relationship between CA125 and hepatitis C. However, no relationship between tumor marker CA19-9 and hepatitis B and $\mathrm{C}$ was observed.

Our findings showed that there is a significant relationship between serum levels of tumor markers CA15-3 in patients with hepatitis B and of tumor markers CA125 in patients with hepatitis C. But no relationship between CA19-9 and hepatitis patients was found.

We revealed that a high AST, ALT, AST/ALT ratio, low platelet count and level CA15-3 and CA125 correlated with severe liver damage. Liver cells were found to be damaged or inflamed, an increased natural range of chemicals such as enzymes into the blood stream to the liver, resulting in an increased amount of enzymes in the blood of the liver. In our study, the low platelet count was correlated with elevated CA125 and CA15-3.

This study found no significant relationship between location, job, sex, albumin, ferritin, ALP, PT and tumor markers. These results are also consistent with studies of El Bokl et al. and Kobeisy et al. [10,11].

The results of the present study about tumor marker CA15-3 have been consistent with the research conducted by Heydarpour et al. Nonetheless, for tumor markers CA125 and CA19-9 they have not been consistent [12]. Our data indicates that serum CA15-3 levels may be elevated in patients with hepatitis. The serum concentration of CA15-3 should thus be considered when severe liver fibrosis is suspected. In a study carried out by El Bokl et al. there has been a significant correlation between CA125 and infection with hepatitis $C$ which was in line with this study [10]. Our study was supported by the findings of Derarbhavi et al. because levels of CA125 were also elevated in their patients [13]. CA125 is not a tissue or tumor specific antigen, thus it can be found in patients with benign and neoplastic pathologies [14]. Our study does not confirm by Bertino et al. who reported that CA19-9 levels in chronic hepatitis and related cirrhosis was statistically significant [15]. A number of studies showed that correlation between CA19-9 and some standard parameters of hepatic function. Some investigation showed that serum CA19-9 levels elevated in hepatocellular, colorectal, esophageal, lung and ovarian carcinomas. The use of CA19-9 as a non-invasive marker for disease activity in patients with hepatitis is not useful. Elevation CA19-9 is not specific for viral hepatitis. Further study seemed to be necessary because a high percentage of false positives and false negative can occur.

Tumor marker increase is certainly not a sign of malignancy in this patient group and these tumor markers are, in fact, independent risk factors for patients. The survival of the patients strongly depends on the damage degree due to liver cancer and the presence of infections HBV and HCV. Considering the limited studies on tumor markers and infection of people with hepatitis $B$ and $C$ both in the world and in Iran, it is recommended that future studies take account of a greater number of patients and a longer period of time in order to obtain more desirable results. Tumor markers can increase frequently in patients without malignant transformation. The causes for the association of tumor markers with liver diseases vary. Elevated CA125 in hepatitis may be independent of etiology. In conclusion, our study suggests that the main cause for the increase of serum CA125 in various benign liver diseases indicates the presence of cirrhosis, therefore proving that this protein can arise in both malignant and benign tumors. It can mostly be used to monitor patients with a known cancer (malignancy) or patients that are suspected of having a tumor [16].

Further study seemed to be necessary due to a high percentage of false positives and false negative occurred. This study can carry out as a cohort with a larger sample size. More research is needed for hepatitis and the sensitivity of these tumor markers should be considered, and maybe combining two tumor markers provide a better characteristic in diagnosis and differentiation of hepatic fibrosis that this will require more research. In the study done by Schoniger-Hekele et al. combined tumor markers CA19-9 and CA125 in liver diseases patients are useful for identifying patients with advanced disorders with high specificity. Patients without combined elevation still require a histological examination to identify severe liver fibrosis [17].

\section{Acknowledgements}

The authors thank the cooperation of Islamic Azad University of Lahijan, the Liver and Gastroenterology Research Center personnel, and the personnel of research and production complex of Zist Faravard Pars. Also we acknowledge all people who provided help in this research. This research is concerning MSc thesis in Islamic Azad University, Lahijan, number 20230507912001, which has been carried out by authors private expenses.

\section{Authors' Contributions}

All authors had equal role in design, work, statistical analysis and manuscript writing.

\section{Funding/Support}

Islamic Azad University, Lahijan Branch.

\section{References}

1. Dennis LK, Eugene B, Stephen H. Harrison' sprinciples of internal medicine. $16{ }^{\text {ed }}$.New York: McGraw-Hill; 2004.

2. Cohan N, Zandieh T, Samiei SH. The prevalence and clinical significance hepatitis B and C co-infection. Iran J Sci. 2006;31(3):156-9. 
3. Lok A, McMahon BJ. Practice guidelines committee, American association for the study of liver diseases and chronic hepatitis B. Hepatology. 2001;34(6):1225-41.

4. Massarat MS, Tahaghoghimehrizi S. Iranian national health survey: a brief report. Arch Iran Med. 2002;5(2):73-9.

5. Johnson RJ, Couser WG. Hepatitis B infection and renal disease: clinical, immunopathogenetic and therapeutic considerations. Kidney Int. 1990;37(2):663-76.

6. Shi J, Su Q, Zhang C, Huang G, Zhu Y. An intelligent decision support algorithm for diagnosis of colorectal cancer through serum tumor markers. Comput Methods Programs Biomed. 2010;100(2):97-107.

7. Gadducci A, Tana R, Cosio S, Genazzani AR. The serum assay of tumour markers in the prognostic evaluation, treatment monitoring and follow-up of patients with cervical cancer: a review of the literature. Crit Rev Oncol Hematol. 2008;66(1):10-20.

8. Motoo Y, Satomura Y, Mouri I, Mouri H, Ohtsubo K, Sakai J, et al. Serum levels of pancreatitis-associated protein in digestive diseases with special reference to gastrointestinal cancers. Dig Dis Sci.1999;44(6):1142-7.

9. Maestranzi S, Przemioslo R, Mitchell H, Sherwood RA. The effect of benign and malignant liver disease on the tumour markers CA19-9 and CEA. Ann Clin Biochem. 1998;35( Pt 1):99-103.

10. El Bokl M, Mabrouk S, Adel Abd El-Mageed NA, Helmy Mahmoud N, Shabana SS. Do serum CA 19-9 and CA 125 levels predict the severity of HCV-related liver fibrosis? Egypt Liver J. 2011;1(1):33-7.

11. Kobeisy MA, Morsy KH, Galal M, Sayed SK, Ashmawy MM, Mohammad FM. Clinical significance of elevated alpha-foetoprotein (AFP) in patients with chronic hepatitis $C$ without hepatocellular carcinoma in upper EGYPT. Arab J Gastroenterol. 2012;13(2):49-53.

12. Heydarpour M, Tavakkoli H, Shafiei D, Koleini N, Arjmandpour A. Correlation between the level of the tumor markers with the stage of liver fibrosis in patients with chronic hepatitis and cirrhosis. Isfahan Med Sch J. 2011;29(155):1239-46.

13. Devarbhavi H, Kaese D, Williams AW, Rakela J, Klee GG, Kamath PS. Cancer antigen 125 in patients with chronic liver disease. Mayo Clin Proc. 2002;77(6):538-41.

14. Canney PA, Moore M, Wilkinson PM, James RD. Ovarian cancer antigen CA125: a prospective clinical assessment of its role as a tumour marker. Br J Cancer. 1984;50(6):765-9.

15. Bertino G, Ardiri AM, Boemi P, Bruno CM, Valenti M, Mazzarino MC, et al. Meaning of elevated CA 19-9 serum levels in chronic hepatitis and HCV-related cirrhosis. Minerva Gastroenterol Dietol. 2007;53(4):305-9.

16. Molina R, Filella X, Bruix J, Mengual P, Bosch J, Calvet X, et al. Cancer antigen 125 in serum and ascitic fluid of patients with liver diseases. Clin Chem. 1991;37(8):1379-83.

17. Schoniger-Hekele M, Muller C. The combined elevation of tumor markers CA 19-9 and CA 125 in liver disease patients is highly specific for severe liver fibrosis. Dig Dis Sci. 2006;51(2):338-45. 